\title{
Distribution of Rest Periods Between Electrically Generated Contractions in Denervated Muscles of Rats
}

\author{
*Douglas E. Dow, *John A. Faulkner, and *†Robert G. Dennis \\ *Institute of Gerontology, Department of Biomedical Engineering, University of Michigan, Ann Arbor, MI; and †Department \\ of Biomedical Engineering, University of North Carolina at Chapel Hill, Chapel Hill, NC, U.S.A.
}

\begin{abstract}
Stimulation protocols for denervated muscles distribute the generated contractions either within treatment sessions followed by hours of rest, or repeated $24 \mathrm{~h}$ per day with each contraction followed by a constant interval of rest. Our purpose was to directly compare the effects of the same number of identically generated contractions having different temporal daily distributions. For 5 weeks in denervated extensor digitorum longus muscles of rats, between 100 and 800 contractions were generated daily, distributed either within worksets that alternated
\end{abstract}

periods of activity and rest, or separated by constant intervals of rest. Most of the tested protocols maintained muscle mass and maximum force near values of innervated controls. Although 100 contractions daily generated at constant intervals were sufficient to maintain mass and force, 100 contractions during a 4-h treatment session followed by $20 \mathrm{~h}$ of rest were not sufficient, and mass and force were not different from values of denervated muscles. Key Words: Extensor digitorum longusActivity-Contractility-Depolarization-Workset.
The contractile and cellular properties of skeletal muscles are influenced by the amount and pattern of contractile activity (1). Denervated muscles undergo no contractile activity, and rapidly lose muscle mass, maximum force, and even specific force that is normalized for the physiological cross-sectional area (CSA) of the muscle (2). We have developed an implantable stimulator (3) and a protocol of electrical stimulation for denervated extensor digitorum longus (EDL) muscles of rats that maintains muscle mass, maximum force, specific force, and mean fiber CSAs at values close to those for control muscles after 5 weeks of stimulation (4). This protocol generates short, tetanic contractions separated by a constant interval of rest, repeated $24 \mathrm{~h}$ per day. In this earlier study, the effect of the distribution of the generated contractions and periods of rest was not studied.

Mammals have periods of more or less intense muscular activity related to the 24-h circadian cycle. Exercise training alters contractile activity and is

Received February 2005.

Address correspondence and reprint requests to Dr. Robert G. Dennis, Department of Biomedical Engineering, University of North Carolina at Chapel Hill, Chapel Hill, NC, 27599, U.S.A. E-mail: bob@unc.edu accomplished within the 24-h cycle that includes long periods of rest. In resistance training, the timing of the periods of rest between contractions or sets of contractions affects fat-free mass, maximum force, and power output (5). For training of muscles in paraplegics with electrical stimulation, muscle force was affected by the length of the treatment session, the ratio of work to rest periods within the treatment session, and the number of treatment sessions per week (6). The timing of the periods of rest between contractions or sets of contractions may also affect electrically stimulated denervated muscles. Certain stimulation-denervation studies grouped all electrically generated contractions for one day into one or more treatment sessions, and those sessions were repeated a number of times per week $(7,8)$. Other studies separated each contraction by a constant interval of rest, repeated $24 \mathrm{~h}$ per day, 7 days per week $(3,4,9)$. Our purpose was to directly compare the effects of the same daily number of identically generated contractions that had different temporal distributions of the rest periods between contractions.

\section{MATERIALS AND METHODS}

The experiment utilized 63 adult male WI/ HicksCar rats (Harlan, Indianapolis, IN, U.S.A.). All 
procedures were conducted in accordance with the guidelines established in the US Public Health Service Guide for the Care of Laboratory Animals and with the approval of the University Committee on the Use and Care of Animals.

Rats were assigned to one of the following three groups (1): denervated ( $n, 5$; final age, $6.1 \pm 0.3$ month; weight, $373 \pm 10 \mathrm{~g}$ ), (2) constant intervals stimulated-denervated $(n, 31$; final age, $6.8 \pm 0.1$ month; weight, $382 \pm 4 \mathrm{~g}$ ), or (3) workset intervals stimulated-denervated $(n, 28$; final age, $7.2 \pm 0.4$ month; weight, $381 \pm 5 \mathrm{~g}$ ). The EDL muscle of the right hindlimb of each rat underwent the experimental interventions involving denervation and stimulation. The left EDL muscle of each rat remained unoperated and innervated, received no stimulation prior to final evaluation, and acted as a control muscle (4). An initial surgery permanently denervated the right EDL muscle in each rat by resection of the sciatic nerve. Immediately following, rats in either of the stimulated-denervated groups had a stimulator (3) and electrode wires (Teflon-coated stainless steel, 36 AWG; AS633, Cooner Wire, Chatsworth, CA, U.S.A.) implanted as shown in Fig. 1 according to the procedure of Dow et al. (4). After a 5-week period following the initial surgery, the denervated or stimulated-denervated muscles were removed and evaluated.

To generate each tetanic contraction, the implanted stimulator generated 20 bipolar pulses (balanced square-wave, $0.2 \mathrm{~ms}$ pulse width for each half pulse) at $100 \mathrm{~Hz}$, with $8 \mathrm{~V}$ pulse amplitude that generated a current of 6-12 mA during each halfpulse $(3,4)$. The number of contractions generated each day $\left(C_{\mathrm{d}}\right)$ was set at a value between 100 and 800 .

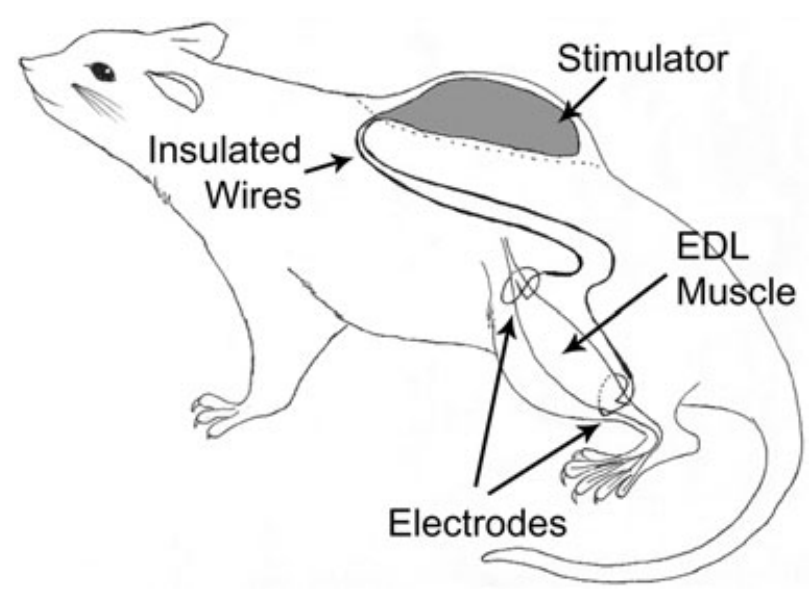

FIG. 1. Placement of the battery powered stimulator implanted under the skin of the rat and electrodes looped around the denervated EDL muscle (Illustration by Megumi Hayashida).

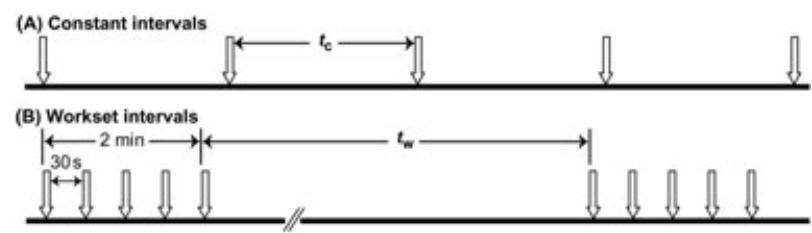

FIG. 2. Distribution of the periods of rest between muscle contractions for protocols of $(A)$ constant intervals or $(B)$ workset intervals. Each down-arrow symbol represents a train of 20 pulses at $100 \mathrm{~Hz}$ to generate one tetanic contraction. In constant intervals $(A)$, each contraction was separated by exactly the same period of rest $\left(t_{c}\right)$ throughout each period of $24 \mathrm{~h}$. In each set of workset intervals (B), 5 contractions were separated by $30 \mathrm{~s}$ of rest, followed by a longer period of rest $\left(t_{w}\right)$. These worksets of 5 contractions followed by $t_{\mathrm{w}}$ of rest were repeated for a 4-h period designated a workout.

Figure 2 contrasts the two types of distributions of contractions and rest periods over each 24-h period. In the constant-intervals distribution, each individual contraction was separated by an equal period of rest $\left(t_{\mathrm{c}}\right)$, and this was repeated $24 \mathrm{~h}$ per day (Fig. 2A). The value of $t_{\mathrm{c}}$ was determined by the desired value of $C_{\mathrm{d}}$, and ranged from $14.4 \mathrm{~min}$ for $C_{\mathrm{d}}$ of 100 to $1.8 \mathrm{~min}$ for $C_{\mathrm{d}}$ of 800 contractions daily.

In the workset-intervals distribution, the generated contractions were distributed into layered sets of work and rest. Five contractions, each separated by $30 \mathrm{~s}$ of rest, were generated during a set (Fig. 2B). Then, a longer period of rest $\left(t_{\mathrm{w}}\right)$ was allowed before the next set began (Fig. 2B, Table 1). These sets of five contractions followed by $t_{\mathrm{w}}$ minutes of rest were repeated throughout a 4-h period called a workout. Each 4-h workout was followed by $2 \mathrm{~h}$ of rest during which no contractions were generated. This allowed a maximum of 4 workouts per day. Figure $3 \mathrm{~A}$ shows a protocol having 4 workouts per day, designated WWW. Figure 3B shows a protocol, designated WRRR, having only 1 workout per day with rest over the remaining $20 \mathrm{~h}$. Table 1 lists the values for $C_{\mathrm{d}}, t_{\mathrm{w}}$, pattern of workouts, and maximum period of continuous rest between contractions during each 24-h day.

TABLE 1. Protocols of workset intervals

\begin{tabular}{lrlc}
\hline$C_{\mathrm{d}}$ & $t_{\mathrm{w}}$ & Pattern & Max rest (h) \\
\hline 100 & 10.0 & WRRR & 20 \\
200 & 10.0 & WRWR & 8 \\
300 & 10.0 & WWWR & 8 \\
400 & 10.0 & WWWW & 2 \\
600 & 4.0 & WWWR & 8 \\
800 & 4.0 & WWWW & 2 \\
\hline
\end{tabular}

Notes: $C_{\mathrm{d}}$ is the number of tetanic contractions generated each day, $t_{\mathrm{w}}$ is defined in Fig. 2B, and Pattern is shown in Fig. 3. 
(A) Four workouts per day (WwWw)

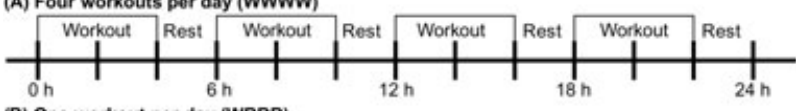

(B) One workout per day (WRRR)

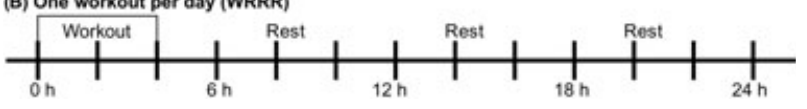

FIG. 3. Examples of workout patterns. Depending on the proto$\mathrm{col}$ of workset intervals, each 6-h period of the 24-h day may have had either a workout lasting $4 \mathrm{~h}$ followed by $2 \mathrm{~h}$ of rest (designated $\mathrm{W}$ ), or a full $6 \mathrm{~h}$ of rest (designated $\mathrm{R}$ ). (A) shows a WWWW pattern, where each 6-h period contained a 4-h workout (W), each followed by $2 \mathrm{~h}$ of no stimulation. (B) shows a WRRR pattern, where the first 6-h period contained a 4-h workout (W) followed by $2 \mathrm{~h}$ of no stimulation, and the other three 6-h periods contained no workouts, only rest $(\mathrm{R})$, resulting in a daily rest period of $20 \mathrm{~h}$ having no contractions.

Following the 5-week treatment period, each rat was anesthetized and the stimulator was checked with use of an oscilloscope for proper functioning. A stimulator was considered defective if the pulses were mono-polar, if the pulse width was longer than $0.6 \mathrm{~ms}$, if the voltage amplitude was less than $5 \mathrm{~V}$, or if the current was less than $1 \mathrm{~mA}$. Physiological data recorded from a muscle with a defective stimulator were discarded without further analysis. After checking the stimulator, the EDL muscles were removed and evaluated according to the procedure of Faulkner et al. $(2,3)$ to determine muscle mass and maximum force for isometric, tetanic contractions. Specific force was normalized for the physiological CSA of the muscle.

Statistical analysis was performed using SPSS software (SPSS Inc., Chicago, IL, U.S.A.). For the dependent variables of muscle mass, maximum force, and specific force, a one-way ANOVA was used to compare differences between the different experimental groups. When a significant main effect was found, the Bonferroni $t$-test was used for post hoc analysis and the 0.05 level of probability was used to signify statistical significance. All data are presented as means \pm standard error (SE).

\section{RESULTS}

Of the 63 rats utilized for this experiment, data were discarded for the right EDL muscles from 5 of the 30 rats with constant intervals and 5 of the 28 rats with workset intervals because the stimulator had become defective as determined by the procedure described above. Data were also discarded for 3 of the 63 left control EDL muscles because the muscle evaluation procedure was inadvertently not completed correctly. Of the 53 implanted stimulators, $17 \%$ became defective prior to final evaluation.
The EDL muscle of rats maintained only $36 \%$ of muscle mass, $9 \%$ of maximum force $\left(P_{\mathrm{o}}\right)$, and $25 \%$ of specific force $\left(s P_{\mathrm{o}}\right)$ after 5 weeks of denervation (Fig. 4). Denervation not only induced atrophy in the muscle, but the remaining muscle tissue was weaker, generating lower values of force, even when normalized by the smaller CSA of the atrophied muscle (Fig. 4A). In contrast, if stimulated with either of the protocols having $C_{\mathrm{d}}$ of between 200 and 800 contractions daily, values for these properties were maintained close to those for control muscles, such that mass was above $90 \%$, and both $P_{\mathrm{o}}$ and $s P_{\mathrm{o}}$ were above $75 \%$ (Fig. 4). For the tested protocols having $C_{\mathrm{d}}$ of 200-800, mass, $P_{\mathrm{o}}$, or $s P_{\mathrm{o}}$ were not different whether the contractions were separated by constant intervals or workset intervals (Fig. 4). In contrast, at $C_{\mathrm{d}}$ of 100 the constant intervals maintained $95 \%$ of mass, $80 \%$ of $P_{\mathrm{o}}$, and $85 \%$ of $s P_{\mathrm{o}}$, but the workset intervals maintained only $53 \%$ of mass, $11 \%$ of $P_{\mathrm{o}}$, and $18 \%$ of $s P_{\mathrm{o}}$. These low values for the workset intervals $\left(C_{\mathrm{d}}, 100\right)$ were not different than values for

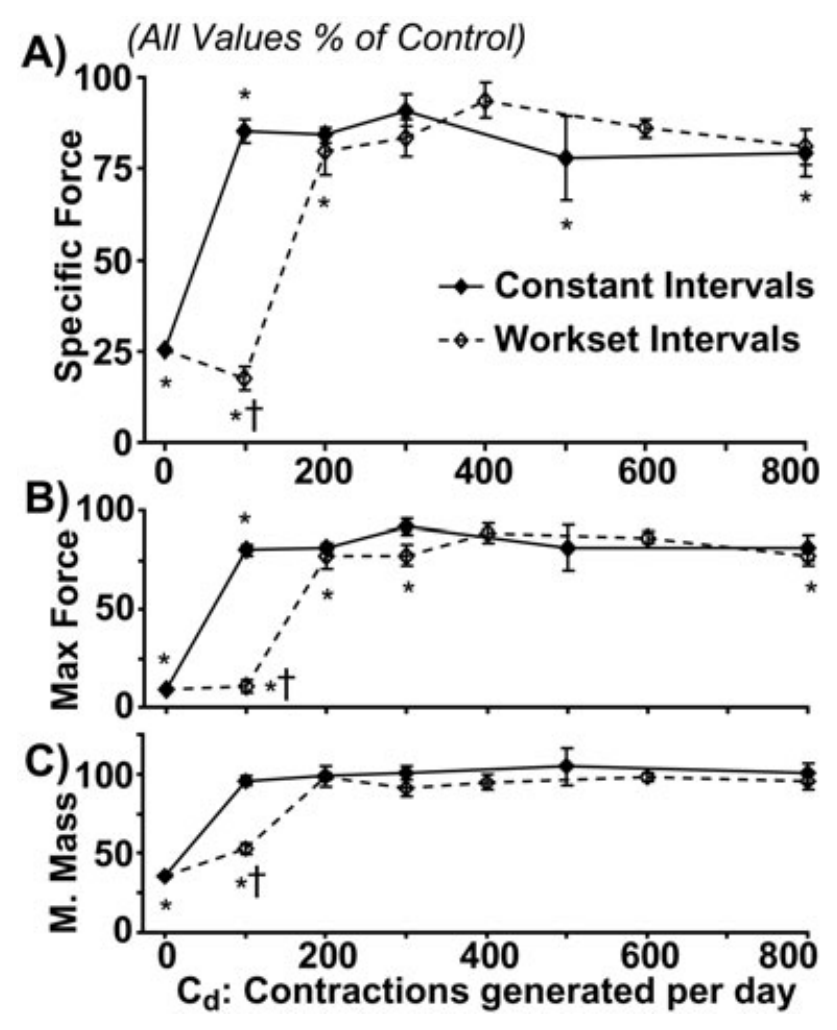

FIG. 4. Specific force (A), maximum force (B), and muscle mass (C) following 5 weeks of denervation and stimulation for different numbers $\left(C_{\mathrm{d}}\right)$ and distributions (constant intervals or worksets) of contractions generated daily. A $C_{d}$ of 0 plots the outcome of denervated muscles that received no stimulation. Error bars are $\mathrm{SE}$. The * indicates difference with values of control muscles $(P<0.05)$. For the workset intervals, the $\dagger$ indicates difference with values of the constant intervals having equal $C_{\mathrm{d}}$ contractions daily $(P<0.05)$. 
denervated muscles that had received no electrical stimulation (Fig. 4).

\section{DISCUSSION}

The maintenance of mass and force were influenced not only by the number of electrically generated contractions, but also by the distribution of these contractions throughout the day (Fig. 4). One difference between the protocols that did maintain mass and force, and the protocol that did not was the maximum continuous period of rest between contractions during each 24-h day. Of the protocols that did maintain mass and force, the constant intervals at $C_{\mathrm{d}}$ of $100 \mathrm{had}$ a maximum rest of $0.24 \mathrm{~h}$, the workset intervals at $C_{\mathrm{d}}$ of 400 and 800 had maximum rest of $2 \mathrm{~h}$, and the workset intervals at $C_{\mathrm{d}}$ of 200, 300, and 600 had maximum rest of $8 \mathrm{~h}$ (Table 1). The workset intervals at $C_{\mathrm{d}}$ of 100 that failed to maintain mass and force had a maximum period of continuous rest each day of $20 \mathrm{~h}$ (Table 1, Figs. 3 and 4). Generation of 100 contractions per day was sufficient to maintain mass and force, but a daily distribution having an excessively long period of rest $(20 \mathrm{~h})$ each day made the protocol insufficient to maintain mass and force.

An overly long period of rest between contractions may be enough to initiate changes in the regulation of gene and protein expression that leads to denervation atrophy in skeletal muscle. Action potentials and the resulting contractions are key regulators of denervation atrophy $(9,10)$. Following the onset of denervation, action potentials from the nerve and resulting contractions cease, and Eftimie et al. have shown that the expression of specific molecular signals changes within hours: myogenin mRNA is upregulated within $8 \mathrm{~h}$ and MyoD within $16 \mathrm{~h}$ (11). Thereafter, myogenin is down-regulated within $4 \mathrm{~h}$ of the onset of electrical stimulation (12). An excessively long period of rest between contractions may initiate the cascade of changes in mRNA and protein expression levels that initiates the processes of denervation atrophy. Further research will be required to determine the maximum period of rest during each 24-h period that would not hinder maintenance of muscle mass and force.

Acknowledgments: This work was supported by NIH grant PO1-AG10821, NIA training grant AG00114-17, and NIDCR training grant DE07057. The authors thank Cheryl A. Hassett for surgical procedures.

\section{REFERENCES}

1. Fitts RH. Effects of regular exercise training on skeletal muscle contractile function. Am J Phys Med Rehabil 2003;82:32031.

2. Carlson BM, Billington L, Faulkner J. Studies on the regenerative recovery of long-term denervated muscle in rats. Restor Neurol Neurosci 1996;10:77-84.

3. Dennis RG, Dow DE, Faulkner JA. An implantable device for stimulation of denervated muscles in rats. Med Eng Phys 2003;25:239-53.

4. Dow DE, Cederna PS, Hassett CA, Kostrominova TY, Faulkner JA, Dennis RG. Numbers of contractions to maintain mass and force of a denervated rat muscle. Muscle Nerve 2004;30:77-86.

5. Kraemer WJ, Ratamess N, Fry AC, et al. Influence of resistance training volume and periodization of physiological and performance adaptations in collegiate women tennis players. Am J Sports Med 2000;28:626-33.

6. Petrofsky JS, Stacy R, Laymon M. The relationship between exercise work intervals and duration of exercise on lower extremity training induced by electrical stimulation in humans with spinal cord injuries. Eur J Appl Physiol 2000;82:504-9.

7. Salerno GM, Bleicher JN, Stromberg BV. Blink reflex recovery after electrical stimulation of the reinnervated orbicularis oculi muscle in dogs. Ann Plast Surg 1990;25:360-71.

8. Kern H, Hofer C, Strohhofer M, Mayr W, Richter W, Stohr H. Standing up with denervated muscles in humans using functional electrical stimulation. Artif Organs 1999;23:447-52.

9. Gundersen K, Eken T. The importance of frequency and amount of electrical stimulation for contractile properties of denervated rat muscles. Acta Physiol Scand 1992;145:49-57.

10. Buffelli M, Pasino E, Cangiano A. Paralysis of rat skeletal muscle equally affects contractile properties as does permanent denervation. J Muscle Res Cell Motil 1997;18:683-95.

11. Eftimie R, Brenner HR, Buonanno A. Myogenin and MyoD join a family of skeletal muscle genes regulated by electrical activity. Proc Natl Acad Sci USA 1991;88:1349-53.

12. Neville CM, Schmidt M, Schmidt J. Response of myogenic determination factors to cessation and resumption of electrical activity in skeletal muscle: a possible role for myogenin in denervation supersensitivity. Cell Mol Neurobiol 1992;12:51127. 